\title{
A GATED ION SOURCE FOR TIME-OF-FLIGHT SPECTROSCOPY
}

\author{
R. H. DAY and W. C. PARKINSON \\ Cyclotron Laboratory, Department of Physics, The University of Michigan, \\ Ann Arbor, Michigan 48105, U.S.A.
}

Received 28 February 1973

A gated ion source using tetrode geometry and having $100 \%$ gating efficiency for all ion species is described. Static transfer characteristics and gating characteristics are presented, and the

\section{Introduction}

Spectroscopy by time-of-flight with good resolution in energy requires short pulses of beam current on the target, with the pulses separated appropriately in time. In the modern isochronous cyclotron the accelerated beam is usually adjusted to have a phase width of the order of a few electrical degrees of the rf cycle in order to obtain high extraction efficiency with good beam quality ${ }^{1}$ ). While this leads to pulses of width of the order of a nanosecond and suitable for time-of-flight measurements, the repetition rate corresponding to the rf cyclotron frequency, normally in the range 5-20 $\mathrm{MHz}$, is too high since particles produced in the target from one cycle can arrive at the detector at essentially the same time as slower particles from preceding cycles. This is particularly true for the long flight paths required to obtain good resolution for energies in the effect of other charge states on the space charge limited current of a given ion species is discussed.

range $10-30 \mathrm{MeV}$. Over the years several schemes have been used to reduce the repetition rate. In the early days $^{2}$ ) of slow neutron spectroscopy the arc-ion source was pulsed, but as is well known the quenching time of the ionization in a gas discharge is of the order of a few microseconds. Electrostatic deflection of the external beam has frequently been used $^{3}$ ), but for the higher particle energies now of interest this requires relatively large electric sweeping fields, and suffers the more telling disadvantage that the unwanted beam pulses are discarded after acceleration to full energy. By discarding them at the center of the cyclotron, the average value of the internal circulating beam and thus the radiation background and build-up of radioactivity in the machine is reduced. One scheme ${ }^{4}$ ) deflects the unwanted beam pulses in the axial direction on the first cyclotron orbit. This requires that deflection plates with

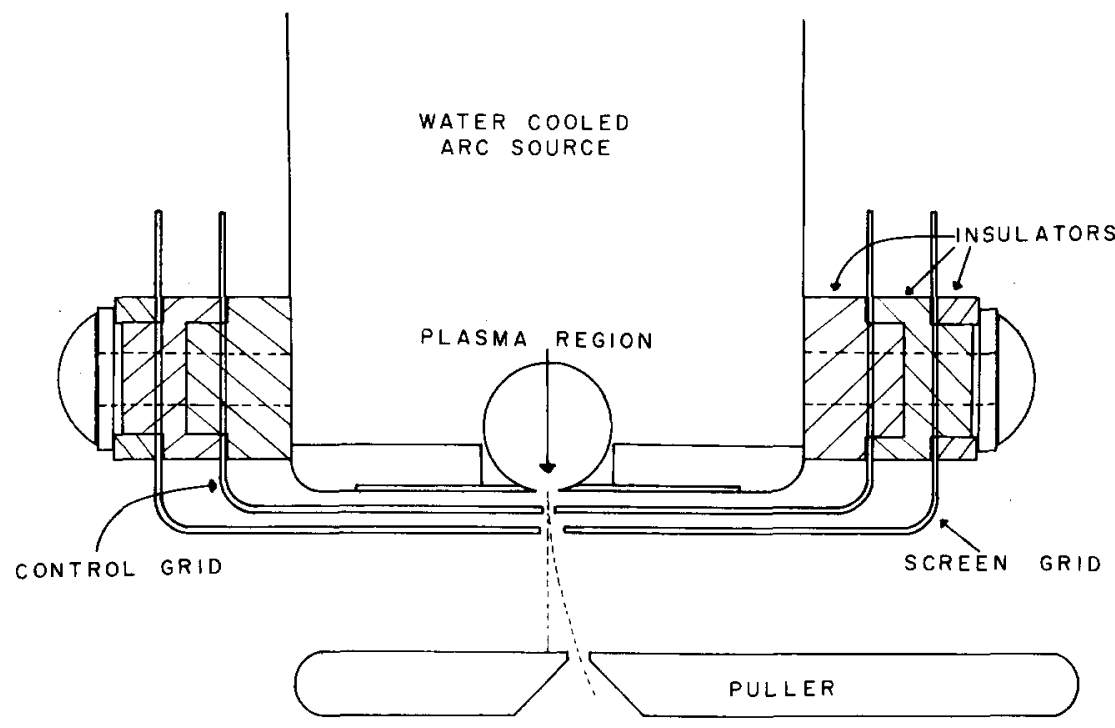

Fig. 1. The electrode structure of the gated ion source to scale. See text for dimensions. 
$\mathrm{dc}$ and ac potentials in the $10 \mathrm{kV}$ range be inserted into the central region. An alternate scheme used by Bently et al..$^{5}$ ) gates the beam emitted from the ion source by inserting a gating electrode in the ion source aperture. In their scheme the current and the gating efficiency depend on the conditions of the ion plasma; in particular successful operation of the gate required low power arc conditions, and $\mathrm{He}^{++}$was not successfully gated.

\section{Ion source geometry}

We have constructed ${ }^{6}$ ) a gated ion source which is similar in principle to that of Bently et al. ${ }^{5}$ ) but which circumvents the disadvantages; the required gating voltage and the efficiency for the extraction of ions from the plasma are essentially independent of dee voltage, and ions of any charge state can be gated with an efficiency of $100 \%$. The design, in analogy with vacuum tubes, corresponds to a tetrode rather than triode geometry. The electrode structure is shown in fig. 1. It consists of the conventional nongated ion source and "puller" with two plane-parallel electrodes, the control "grid" and the screen "grid", interposed between them. The control grid and screen are formed from tantalum sheet $0.010^{\prime \prime}$ thick. Each electrode has a single slit, and the slits are aligned on the trajectory of the accelerated ions as shown in fig. 1. Thus the electrodes are not in fact grids; the word as used here is in the parlance of vacuum tubes. Typical dimensions of the slits used for both singly and doubly ionized ions ( $\mathrm{d}$ and ${ }^{3} \mathrm{He}^{++}$) are $0.5^{\prime \prime}$ high by $0.040^{\prime \prime}, 0.020^{\prime \prime}, 0.040^{\prime \prime}$ and $0.040^{\prime \prime}$ for the cathode, control grid, screen grid and puller,

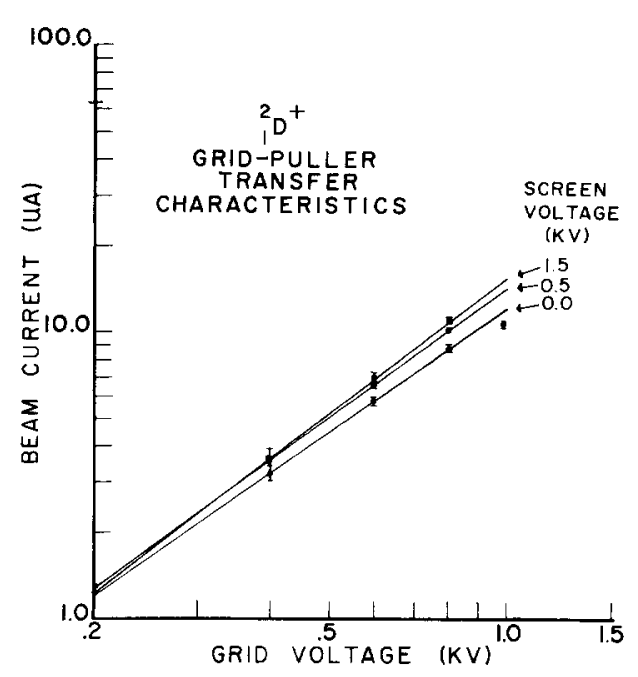

Fig. 2. The grid-puller transfer characteristics for ${ }_{1}^{2} \mathrm{D}^{+}$with the screen potential as a parameter. The slopes vary from 1.42 to 1.58 . respectively. The interelectrode distances are $0.040^{\prime \prime}$ cathode to control grid, $0.031^{\prime \prime}$ grid to screen and $0.312^{\prime \prime}$ screen to puller. The function of the screen grid is to shield the control grid and cathode from the electric field of the puller. The plasma in the ion tower (the cathode) is caused to develop at the position of and parallel to the cathode slit. In the normal ungated mode of operation, negative dc bias voltages are applied to both the control and the screen grids. The puller is attached to the dee into which the ion beam is injected, thus the puller voltage is the rf dee voltage. In the "gated" mode a negative gating voltage, synchronized with the dee voltage, is applied to the control grid and a constant and negative de voltage is applied to the screen. While a positive de bias voltage in addition to the ac gating voltage could be applied to the control grid to ensure current cutoff, it is found not to be necessary.

\section{Transfer characteristics}

The "static" grid-puller transfer characteristics for constant screen and puller voltage with the screen voltage as a parameter are displayed on log-log plots in figs. 2 and 3 . These were measured in The University of Michigan $83^{\prime \prime}$ cyclotron for deuterons and ${ }^{3} \mathrm{He}^{++}$ ions of full energy $11.2 \mathrm{MeV}$ and $22 \mathrm{MeV}$, respectively. The "puller current" is not the beam striking the puller, but rather it is the current passing through the puller slit into the dee. The puller voltage is the rf dee voltage, so that by constant puller voltage we mean the amplitude of the $\mathrm{rf}$ is held constant. For the electrode geometry shown, it is the potential of the control grid rather than the puller that determines the beam current

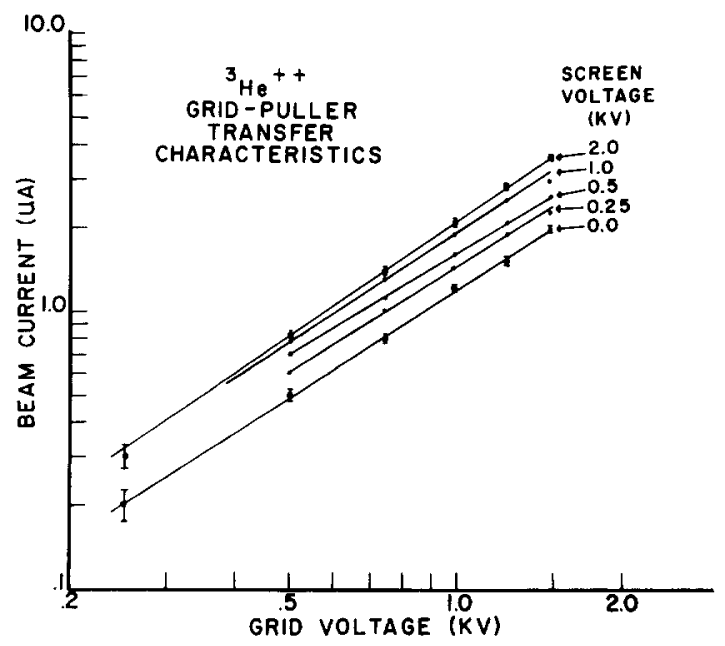

Fig. 3. The grid-puller transfer characteristics for ${ }^{3} \mathrm{He}^{++}$, with the screen potential as a parameter. The slopes vary from 1.20 to 1.35 (see text). 
while the potentials of the three electrodes determine the trajectory of the ions through the electrode structure. The average beam current was measured by means of a current probe in the median plane of the cyclotron after the ions had been accelerated through several turns. The phase width of the pulses of beam current, in degrees of the rf cycle, is determined by the electrode geometry and the dee voltage, and for these measurements was about $20^{\circ}$ fwhm as indicated by a phase probe which is a part of our standard beam diagnostic instrumentation.

The number of positive ions available for extraction from the ion plasma depends on the operating conditions of the gas discharge ${ }^{7}$ ). Under "normal" operating conditions corresponding to a high ion density, the current extracted from the plasma is space charge limited (temperature saturated), while for low ion densities, the current displays voltage saturation, analc gous to temperature limited current in a therm-

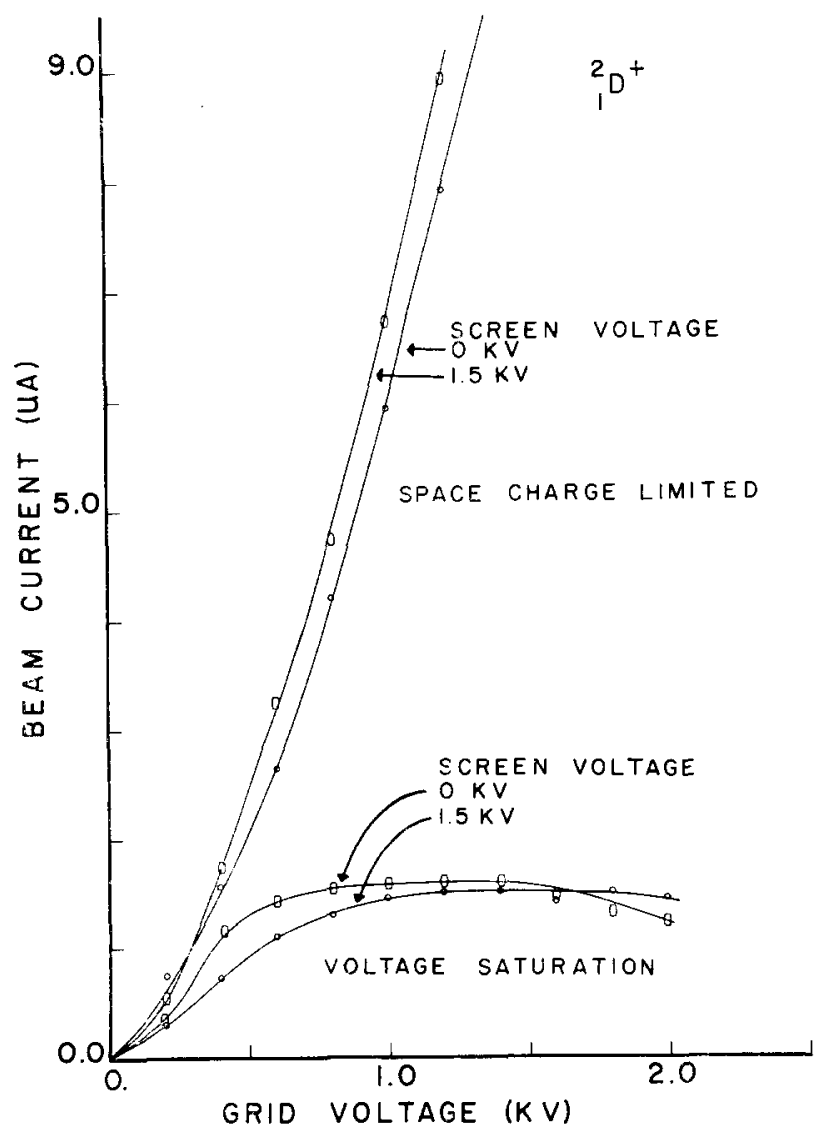

Fig. 4. The grid-puller transfer characteristics for normal operating conditions of the arc source (space charge limited) and for the arc source operated at low current and voltage (voltage saturation). ionic electron tube. The measured characteristics for the two cases are shown in fig. 4 . In both case the current is essentially independent of the puller voltage, indicating that the cathode is shielded from the electric field of the puller. (A slight decrease in current with puller voltage was observed, but this is accounted for by the fact that as the dee voltage is lowered fewer of the lower energy ions in the pulse clear the ion tower structure on the first full turn in the cyclotron.) More direct evidence of the shielding is that the current is zero for zero control grid voltage. In taking these data the puller slit was adjusted parallel to itself to match the ion trajectory (maximize the current) keeping the separation between the puller and screen grid constant. The screen and control grid slits could not be adjusted to match the trajectories as their potentials were changed, and this accounts for the decrease in current at large grid voltages.

\section{Space charge limitations}

The beam current may be expressed in the form $I_{\mathrm{b}}=K V_{\mathrm{c}}^{\alpha}$, where $K$ is a constant and $V_{\mathrm{c}}$ is the controlgrid voltage. From the $\log -\log$ plot of fig. 2 for deuterons the value of $\alpha$ is found to vary from 1.42 to 1.58 depending on the screen potential and from fig. 3 for ${ }^{3} \mathrm{He}^{++} \alpha$ varies from 1.20 to 1.35 . In each case the slope is constant over the full range of grid voltage. The scatter in the data points gives an estimate of the accuracy of the measurements, which is of the order of $\pm 10 \%$. For a plane parallel geometry the space charge limited current should vary as $V^{3 / 2} / d^{2}$ (Childs' Law), where $V$ is the diode potential and $d$ the separation of the electrodes. The data agree as well as expected with Childs' Law over the range of measured currents, particularly since the initial conditions at the cathode must depend on the condition of the plasma. It is interesting to note that for some 22 commercial thermionic diodes the value of $\alpha$ was found to vary ${ }^{8}$ ) over the range 1.24 to 1.49 .

The fact that the current is space charge limited has some interesting consequences for cyclotron ion sources. For the plane parallel gated source, with a gridcathode spacing $d_{\mathrm{g}}$, the control grid potential required to give the same beam current as the normal ion source (cathode-puller spacing $d_{\mathrm{n}}$ ) is reduced in the ratio $\left(d_{\mathrm{g}} / d_{\mathrm{n}}\right)^{4 / 3}$. This agrees well with the measured ratio. (For a cylindrical geometry the factor is less favorable.) Another consequence is that a simple measurement of the beam current allows one to make rough estimates of the population of other charge states in the ion plasma. For a single ion species of charge $e_{i}$ and 
mass $m_{i}$ the current density given by Childs' law is

$$
J=\frac{4}{9}\left(2 e_{i} / m_{i}\right)^{1 / 2} \varepsilon_{0} V^{3 / 2} / d^{2} \mathrm{~A} / \mathrm{m}^{2},
$$

where $\varepsilon_{0}$ is the permittivity of free space. It can be shown that for a mixture of charge states in the plasma, the current density of one of the species is

$$
J_{1}=\frac{4}{9} \varepsilon_{0}\left[\frac{\left(2 e_{i} / m_{1}\right)^{\frac{1}{2}}}{1+\sum_{i=2}^{k} n_{i} e_{i} /\left(n_{1} e_{1}\right)}\right] \cdot \frac{V^{3 / 2}}{d^{2}},
$$

where $n_{i}$ is the charge density in the plasma of ions of species $i$. This follows from the fact that $\operatorname{div} J_{i}+\partial \rho_{i} / \partial t$ $=0$ for each ion species $i$. For the gated source with $100 \%{ }^{3} \mathrm{He}^{++}$and with $d=0.040^{\prime \prime}, V=2.0 \mathrm{kV}$, control grid slit area $=6.45 \times 10^{-6} \mathrm{~m}^{2}$ and phase width of $20^{\circ}$ the expected (dc) current is $1.39 \mathrm{~mA}$, while the measured current is only $5 \mu \mathrm{A}$. This large discrepancy results from two factors. Because of the rf puller voltage, the ions are fanned out across the puller slit. This is estimated to account for a factor of 5 , which leaves a factor of 55 to be accounted for by contributions to the space charge by other charge states of ${ }^{3} \mathrm{He}$. Assuming the only other charge state is ${ }^{3} \mathrm{He}^{+}$, the relative population of ${ }^{3} \mathrm{He}^{++}$in the discharge is about $2 \%$. This is consistent with the measurements of yields of hooded type arc sources by Papineau et al. ${ }^{9}$ ).

For deuterium the expected current is $1.44 \mathrm{~mA}$ which is reduced to $290 \mu \mathrm{A}$ after taking into account the ion trajectories. The measured value is $50 \mu \mathrm{A}$, leaving a factor 6 unaccounted. The dominant charge states in deuterium are ${ }_{1}^{2} \mathrm{D}^{+}$and the molecule ions ${ }_{2}^{2} \mathrm{D}^{+}$and ${ }_{3}^{2} \mathrm{D}^{+}$. (While the ${ }_{3}^{2} \mathrm{D}^{+}$is accelerated on the third harmonic, very little reaches the current probe because it is blocked by slits in the central geometry. When the slits are removed, the measured ratio is about 1 to 1.5 .) The relative population densities (for hydrogen) have been measured to be $1: 2: 1.5$ for a similar ion source ${ }^{9}$ ) which again is of the correct order to account for the discrepancy.

It is well known among cyclotron operators that the beam current depends on optimizing the ion source conditions, that is, optimizing the gas discharge and the geometry. The effect is illustrated in fig. 5 which is a $\log -\log$ plot of the transfer characteristics for a high current gas discharge in deuterium $(2.0 \mathrm{~A}$ and $200 \mathrm{~V}$ anode current and voltage, with a high gas flow of $5 \mathrm{~cm}^{3} / \mathrm{min}$ ). Three discreet values of $\alpha$ are obtained depending on the value of the grid bias and the puller position. At low grid voltage $\alpha$ is approximately 2.0; at intermediate values $\alpha$ is 1.55 . At high grid voltage ( $2 \mathrm{kV}$ and $1.5 \mathrm{kV}$ screen voltage) the two datum points indicate an $\alpha$ of 7.5. This effect may be due to changes in the population densities of the ion species due to changes in the electric field at the plasma interface.

\section{Gated operation}

In the "gated" mode of operation, a gating voltage, either pulse or sine-wave, is applied to the grid. The voltage is manufactured by picking an rf reference signal from the dee, passing it through a variable time delay, and counting down to the desired mode $(f / 2$, $f / 3, \ldots)$ with standard TTL circuitry. The resulting square wave can be clipped and amplified and applied to the control grid through a transmission line incorporated on the ion source tube. Alternatively, and for $f / 2$ and $f / 3$ modes it is more convenient, the square wave is used to drive a tube-type LC-resonant tuned power output stage. The total ion and electron loading on the grid structure is negligible.

The gated circulating beam was extracted from the cyclotron, magnetically analyzed, and focused in the scattering chamber. The gating efficiency was then determined by observing with the phase probe the prompt $\gamma$-rays produced by the gated beam striking a target in the chamber. It is difficult to determine the gating efficiency with the internal circulating beam since all the beam in one turn must be intercepted on that turn. Otherwise, the fraction that misses the probe can make an additional turn before striking the probe, giving rise to $\gamma$-ray pulses that appear to be due to successive turns. Pulses of current observed directly on

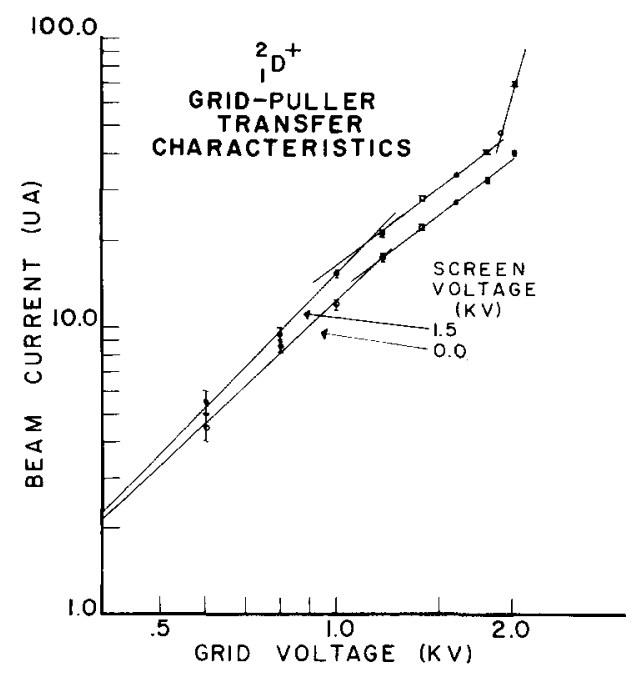

Fig. 5. The grid-puller characteristics for a high current gas discharge in deuterium. The three discrete values of the slopes may be the result of changes in the population densities of the ion species with electric field at the plasma interface. 


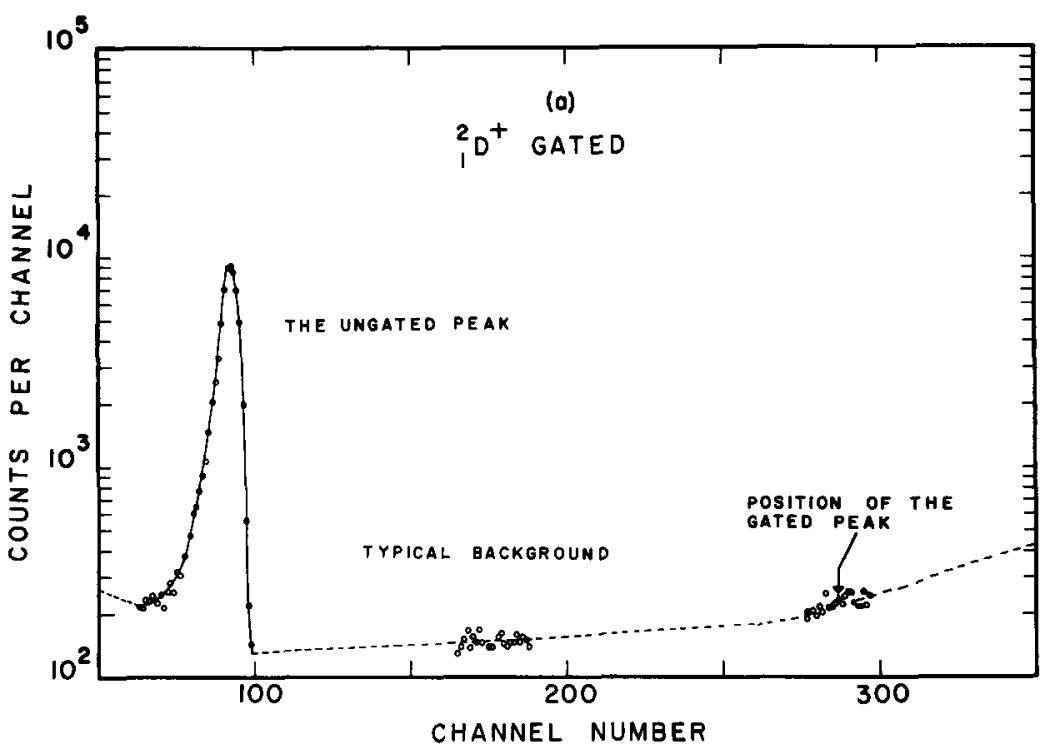

Fig. 6. A gated time-spectrum for ${ }_{1}^{2} \mathrm{D}^{+}$.

an internal probe constructed as a transmission line are even more misleading since beam circulating some distance from the probe induces current pulses in the

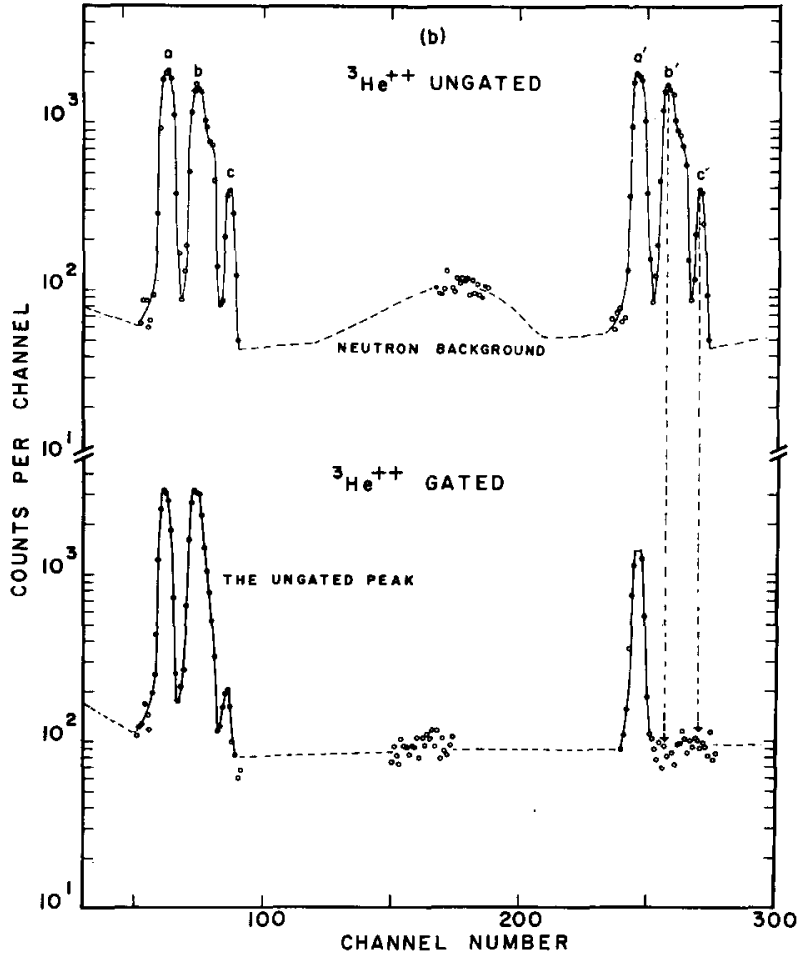

Fig. 7. Ungated and gated time-spectra for ${ }^{3} \mathrm{He}^{++}$. See text for explanation. line. We do not agree with Bently et al. ${ }^{5}$ ) that it is essential to have single turn extraction for measuring gating efficiency, but we do agree that a properly gated ion source is an excellent diagnostic tool for studying the time and space structure of the extracted beam, as illustrated in fig. 6 . The spectrum of prompt $\gamma$-rays and neutrons resulting from the gated deuteron beam and single turn extraction is shown in fig. 6. The gating efficiency is essentially $100 \%$. The rejection ratio is at least $10^{3}: 1$, the limit being determined by the statistics of the background of low energy neutrons produced by the beam pulses on the target. The spectrum of $\gamma$-rays and neutrons from ${ }^{3} \mathrm{He}^{++}$are shown in fig. 7. The three peaks are different slices of the internal beam that meet the criteria for extraction. Peaks $b$ and $c$ are extracted in a single turn with a gating efficiency of at least $4 \times 10^{3}: 1$, the limit again being determined by the background statistics. Peak a, however, is extracted in two turns resulting in counts on every rf cycle.

The electronic gating circuit was designed and constructed by D. DuPlantis and the phase probe assembled and timed by R. Polichar. The mechanical construction was under the supervision of W. E. Downer. It is a pleasure to acknowledge their contributions.

\section{References}

1) W. C. Parkinson and J. Bardwick, Nucl. Instr. and Meth 78 (1970) 245. 
2) See for example, W. W. Havens, Jr. and L. J. Rainwater, Phys. Rev. 83 (1951) 1123.

3) R. Grismore and W. C. Parkinson, Rev. Sci. Instr. 28 (1957) 245; also H. Fauska, R. E. Karns and D. M. Drake, Nucl. Instr. and Meth. 72 (1969) 233.

4) A Svanheden, H. A. Howe and M. Reiser, IEEE Trans. Nucl. Sci. NS-12, no. 3 (1965) 977.

5) R. F. Bently, L. A. Erb, D. A. Lind, C. D. Zafiratos and
C. S. Zaidins, Nucl. Instr. and Meth. 83 (1970) 245.

6) R. H. Day and W. C. Parkinson, Annual Report (Cyclotron Laboratory, The University of Michigan, March 1971).

7) Electrical discharges in magnetic fields (eds. A. Guthrie and R. K. Wakerling; McGraw-Hill, New York, 1949).

8) R. H. Dishington, Elec. Eng. 67 (1948) 1043

$\left.{ }^{9}\right)$ A Papineou, P. Benezech and R. Maillard, J. Phys. Radium $21(1960) 410$. 